\title{
Survey on "Knowledge and Attitudes Regarding the Coronavirus Pandemic" in the Austrian, German and Swiss population
}

Univ.-Prof. Dr. Manuel Schabus ${ }^{1,2} \&$ M. Sc. Esther-Sevil Eigl ${ }^{1,2}$

\footnotetext{
${ }^{1}$ University of Salzburg; Department of Psychology; Laboratory for Sleep, Cognition and Consciousness Research; Salzburg, Austria

${ }^{2}$ University of Salzburg; Centre for Cognitive Neuroscience Salzburg (CCNS); Salzburg, Austria
}

The survey on "Knowledge and Attitudes Regarding the Coronavirus Pandemic" was available online from 17. Jan 2021 to 19. Feb 2021 for the Austrian population. The German version closed a week later (26. Feb 2021). The aim of the study was to obtain a representative overview of the assessment of the current pandemic situation by the German-speaking general population (Austria, Germany, and Switzerland).

\section{Results - Austrian population}

Here data of 3848 adults living in Austria were analysed (64.4\% female, 35.3\% male, 0.3\% diverse). Broad advertisement of the survey in the Austrian media via the Austrian Press Agency (APA) and ORF (Austrian Broadcasting Corporation) as well as a homogeneous age distribution (see Table 1) made it possible to obtain a good and "representative" overview of the current attitudes and state of mind of the Austrian society on the subject of "Corona". Only the 60-69 and 70+ age groups were slightly less represented, with $12.81 \%$ as expected for an online survey.

Table 1. Age distribution of Austrian population in survey

\begin{tabular}{|c|c|c|}
\hline Age-group & $\boldsymbol{n}$ & $\boldsymbol{\%}$ \\
\hline $18-29$ & 723 & $18.79 \%$ \\
\hline $30-39$ & 872 & $22.66 \%$ \\
\hline $40-49$ & 983 & $25.55 \%$ \\
\hline $50-59$ & 777 & $20.19 \%$ \\
\hline $60-69$ & 387 & $10.06 \%$ \\
\hline $70+$ & 106 & $2.75 \%$ \\
\hline Total & $\mathbf{3 8 4 8}$ & $\mathbf{1 0 0 . 0 0 \%}$ \\
\hline
\end{tabular}

In addition, we compared two extreme groups of people in the sample: those who almost exclusively consume public television $(n=874)$ vs. those who also frequently consume private television (and do not consume public television daily, $n=812$ ). Additionally, we compared nonacademic participants $(n=2082)$ vs. academic participants $(n=1766)$. Both comparisons revealed 
contrasting responses, which are explained below as a complement to each section. In contrast, the effects across age and gender were largely equally distributed.

\section{Perceived Restriction \& Public and Private Statements}

$89.2 \%$ of the participants feel ,very“ $(60.4 \%)$ or at least „somewhat“ $(28.8 \%)$ constrained by the Corona-related measures. Most participants (81.5\%) share these concerns/displeasure in private with friends or family ,,regularly“ or „,several times“. Here, all age groups are equally critical. 27.7\% of participants also engage publicly by posting on forums, participating in demonstrations, or even taking legal action. More than one-third of participants (39.2\%) were bothered,,all the time“ or „,most of the time" by feelings of anger and unease as they have the impression that reports are not objective. $57.1 \%$ of graduate participants and $63.2 \%$ of non-graduate participants felt ,very“ constrained by Corona-related actions. Evaluating the same question for the two TV consumption groups reveals a feeling of constrain for $47.7 \%$ of the public TV viewers vs. $72.5 \%$ of the private TV viewers. The groups beyond the age of 60 also feel ,very“ (45.1\%) or "somewhat" (35.3\%) restricted.

Their concerns/displeasure about Corona is shared in private with friends or family by $65.8 \%$ of public TV viewers and $94.4 \%$ of private TV viewers „,regularly“ or ,,several times“. Women and men are equally concerned and critical here, and even $71 \%$ of the $60+$ group express their concerns about Corona-related measures and changes in the law „regularly“ or „several times“ in private.

Feelings of anger and unease due to the impression that reports are not objective preoccupied $31.7 \%$ of academics and $45.5 \%$ of non-academics. Again, viewers of primarily public vs. private television had clearly different views: $10.4 \%$ compared with $68.6 \%$ rated reporting as not objective and neutral ,all the time” or „most of the time“.

\section{Fears}

The greatest fears perceived in the current pandemic are 1) that a close relative will get infected $(67.2 \%), 2)$ the economic damage $(46.9 \%)$ and 3$)$ the restriction of freedom of expression or of fundamental rights (46\%). On the other hand, the fear of actually dying from the coronavirus disease was mentioned least frequently $(15.2 \%)$.

In a comparative analysis based on which TV medium is primarily consumed to gain information about the corona pandemic, opinions once again differed greatly: while $80.2 \%$ of public TV viewers fear that a close relative will be infected, only $45.2 \%$ of private TV viewers do. In terms of restrictions on fundamental rights and freedom of expression, $24.6 \%$ of public TV viewers are worried, compared 
with $76.3 \%$ of private TV viewers. Physical long-term consequences on the other hand are feared by $53.4 \%$ of public TV vs. $21.1 \%$ of private TV viewers. Even suffering from severe symptoms due to Corona is feared by $53.1 \%$ of public vs. $15.9 \%$ of private TV consumers. Actual fear of dying due to Corona is found among $18.1 \%$ of public TV viewers and only $9.1 \%$ of private TV viewers.

Fear of psychological damage is slightly higher among women (38.2\%) than among men (31.4\%) and higher among non-academics (39.4\%) than among academics (32.3\%). Economic damage is feared by every second citizen ( $51.8 \%$ of non-academics and $41.7 \%$ of academics).

\section{Perceived Burdens}

The most worrisome burdens in the pandemic are: 1) not being able to maintain social contacts (78.5\% not being able to meet friends, $78 \%$ not being able to meet relatives), 2) home-schooling for parents $(70.3 \%)$ and 3 ) economic consequences (70.2\%). Even in the $60+$ group, not being able to meet friends or relatives in person is ranked in the top 3 most stressful factors and with $80.3 \%$. Surprisingly, the fear of being a carrier of the disease (45.1\%), of falling ill oneself $(24.4 \%)$ or of a lack of care due to a possible overload of the health care system (44.3\%) is rated as less stressful than the previously mentioned social and economic consequences.

In the gender comparison, $80.6 \%$ of women and $73.4 \%$ of men stated that it was (,,very“ or ,slightly“) burdensome to no longer meet relatives in person on a regular basis. Women $(58.3 \%)$ were more concerned than men (46.2\%) about a close relative becoming ill. Separate analysis by those who primarily consume public vs. those who also regularly consume private television again revealed much greater concern among the former (public TV) group (75.7\% vs. 31.6\%). Personal concern about contracting Corona was also reported to be much higher among public-television viewers $(63.7 \%)$ than among those who in addition regularly watch private television (23.6\%).

Private conflicts due to discussions regarding Corona are much more common among women $(52.6 \%$ vs. $43.4 \%$ for men) and viewers of private TV ( $62.5 \%$ vs. $37.9 \%$ for public TV). Furthermore, women experience the topic of home-schooling as very stressful (74.7\% vs. $61.7 \%$ for the male counterpart). Women also experience hearing/watching news about Corona (65.5\%) as significantly more stressful than men $(52.1 \%)$.

Economic consequences are perceived by women (73\%) as (,,very“) burdensome, and even for 2/3 of the men $(65.3 \%)$. It is interesting to note that, once again, people who regularly watch private television $(84.8 \%)$ are generally significantly more worried than people who mainly watch public television $(57.3 \%)$. 
The same picture is found for the fear of collateral health damage (i.e., a lack of care for other illnesses, postponed surgeries, etc.) with $70.6 \%$ of (,very“) concerned women and $62.3 \%$ of (,,very“) concerned men, and $58.6 \%$ for public TV viewers vs. $79.7 \%$ for those who regularly consume private TV.

When asked about impaired sleep patterns due to the pandemic, more women report problems $(30.4 \%)$ than men $(19.5 \%)$. In general worse perceived sleep quality is found in people who are personally worried by COVID-19 across cultures as we recently published [1].

\section{Estimated Probability of Falling III}

The answer to the question "How likely do you think "Corona" is to cause you a lifethreatening illness (in \%) over the next 12 months?" is also very interesting. Based on all cases already infected with SARS-CoV-2, the statistical probability i) of being hospitalized ranges from $0.25 \%$ (1029 years) to $17.1 \%$ (75-79 years), ii) of ending up in the ICU ranges from $0.24 \%$ (18-29 years) to $2.81 \%(70+)$. [Note: $14 \%$ of hospitalized Covid-19 sufferers receive intensive care on average; source $\mathrm{RKI}]$. Note that the subjectively experienced risk is overestimated 6 -fold $(70+)$ to 58 -fold $(<39)$ in the Austrian population. That is, Austrian citizens (18-69) expect a chance of about 1:10 to need intensive care in a hospital when they get infected by SARS-CoV-2. Scientifically, the more realistic chance of ICU admission is at max. 1 in 400 for age 18-49, and 1:100 for an age up to 69 according to the Swiss database (Table 2). Even lower risks are provided by the QCOVID risk calculator from the University of Oxford (see below) [2] and for England.

Table 2. Risk of hospitalisation and mortality from Covid-19 by age. Data is from the federal office of public health Switzerland.

\begin{tabular}{|c|c|c|c|c|c|c|c|c|c|c|c|c|c|c|}
\hline \multirow{2}{*}{ Age } & \multicolumn{2}{|c|}{ Cases } & \multicolumn{2}{|c|}{ Hospitalisation } & \multirow{2}{*}{$\begin{array}{l}\text { Cases } \\
\text { Hospital }\end{array}$} & \multicolumn{2}{|l|}{ Mortality } & \multirow{2}{*}{ Case fatality } & \multirow{3}{*}{$\begin{array}{l}\text { Hospitalisation by } \\
\text { age }\end{array}$} & \multirow{3}{*}{ Mortality\% by age } & \multirow{3}{*}{$\begin{array}{l}\text { Age groups } \\
\text { survey }\end{array}$} & \multirow{3}{*}{$\begin{array}{l}\text { Probability infected and } \\
\text { ICU }\end{array}$} & \multirow{3}{*}{$\begin{array}{l}\text { Subjective estimate to } \\
\text { come to ICU due to } \\
\text { Covid-19 (1) }\end{array}$} & \multirow{3}{*}{$\begin{array}{l}\text { FACTOR of subjective over- } \\
\text { estimation of risk }\end{array}$} \\
\hline & $\mathrm{N}$ & Incidence & \begin{tabular}{|l|l|}
$N$ & \\
\end{tabular} & Incidence & & N & incidence & & & & & & & \\
\hline $0-9$ & 2037 & 232 & 62 & & $-*$ & 0 & 0 & -7 & & & & & & \\
\hline $10-19$ & 20775 & 2450 & 51 & 6 & $0.2 \%$ & 0 & 0 & $0.0 \%$ & $0,64 \%$ & $0,00 \%$ & & & & \\
\hline $20-29$ & 46321 & $44^{4} 12$ & 137 & 13 & $0.3 \%$ & 0 & 0 & $0.0 \%$ & $1,73 \%$ & $0,00 \%$ & $18-29$ & $0,24 \%$ & $10,22 \%$ & 43 \\
\hline $30-34$ & 22861 & 3720 & 87 & 14 & $0.4 \%$ & 1 & 0 & $0.0 \%$ & $1,10 \%$ & $0,04 \%$ & & & & \\
\hline $35-39$ & 19677 & 3176 & 108 & 17 & $0.5 \%$ & 1 & 0 & $0.0 \%$ & $1,36 \%$ & $0,04 \%$ & 30-39 & $0.17 \%$ & $9,80 \%$ & 58 \\
\hline $40-44$ & 19078 & 3230 & 157 & 27 & $0.8 \%$ & 2 & 0 & $0.0 \%$ & $1,98 \%$ & $0.08 \%$ & & & & \\
\hline $45-49$ & 19917 & 3249 & 242 & 39 & $1.2 \%$ & 6 & 1 & $0.0 \%$ & $3,06 \%$ & $0,23 \%$ & $40-49$ & $0,35 \%$ & $8,99 \%$ & 26 \\
\hline $50-54$ & 20408 & 3064 & 382 & 57 & $1.9 \%$ & 6 & 1 & $0.0 \%$ & $4.83 \%$ & $0,23 \%$ & & & & \\
\hline $55-59$ & 19199 & 3034 & 523 & 83 & $2.7 \%$ & 26. & 4 & $0.1 \%$ & $6,61 \%$ & $0,99 \%$ & 50-59 & 0,80\% & $10,19 \%$ & 13 \\
\hline $60-64$ & 13638 & 2623 & 661 & 127 & $4.8 \%$ & 37 & 7 & $0.3 \%$ & $8,35 \%$ & $1,41 \%$ & $60-69$ & & & \\
\hline $65-69$ & 8764 & 2025 & 742 & 171 & $8.5 \%$ & 103 & 24 & $1.2 \%$ & $9,38 \%$ & $3,92 \%$ & $60-69$ & $1,24 \%$ & $12,56 \%$ & 10 \\
\hline $70-74$ & 7725 & 1922 & 964 & 240 & $12.5 \%$ & 197 & 49 & $2.6 \%$ & $12,18 \%$ & $7,50 \%$ & & & & \\
\hline $75-79$ & 6672 & 2066 & 1144 & 354 & $17.1 \%$ & 357 & 111 & $5.4 \%$ & $14,46 \%$ & $13,60 \%$ & $70+$ & $2,81 \%$ & $16,78 \%$ & 6 \\
\hline $80+$ & 13488 & 2963 & 2654 & 583 & $19.7 \%$ & 1889 & 415 & $14.0 \%$ & $33,54 \%$ & $71,96 \%$ & & & & \\
\hline Total & \begin{tabular}{|l|}
240608 \\
\end{tabular} & 2783 & 7914 & 92 & $3.3 \%$ & 2625 & 30 & $1.1 \%$ & $99,22 \%$ & $100,00 \%$ & Assu & 6 of hospita & sufferers a & sive cat \\
\hline
\end{tabular}

Source: Federal Heath office (Covid-19 vaccination strategy 24.12.20)

Interestingly, however, excess mortality in the total population is not overestimated but rather underestimated. When asked about excess mortality for 2020, 25.8\% still assume a very high or high excess mortality, but then put the excess mortality at 3872 cases on average (trimmed mean 3699; 
95\% confidence interval 3741 - 4003). In fact, however, mortality in 2020 was 5350 cases above the expected variation. Mortality in Austria was 83.386 ( \pm 2791 standard deviation) in 2019, and 91.527 in 2020. (Please note that this excess mortality is unadjusted for the increasing proportion of the 65+ age group in the general population over the years.)

When evaluated separately by sex and primary TV medium, women (18-69yrs) systematically overestimated risk more than men, and more so the younger they are (see Table 3 ).

Table 3. Subjectively estimated probability to be admitted to the intensive care unit (ICU) due to Covid-19 (by gender) vs. the objective risk.

\begin{tabular}{|c|c|c|c|c|c|}
\hline \multirow[t]{2}{*}{$\begin{array}{c}\text { AGE-groups } \\
\text { survey }\end{array}$} & \multirow[t]{2}{*}{$\begin{array}{c}\text { Probability } \\
\text { infected AND } \\
\text { ICU }\end{array}$} & \multicolumn{2}{|c|}{$\begin{array}{l}\text { Subjective estimate to come to ICU due } \\
\text { to Covid-19 (!) }\end{array}$} & \multicolumn{2}{|c|}{ Factor of subjective overestimation of risk } \\
\hline & & Women & Men & Women & Men \\
\hline $18-29$ & $0,24 \%$ & $12,15 \%$ & $6,31 \%$ & 51 & 26 \\
\hline 30-39 & $0,17 \%$ & $11,05 \%$ & $7,59 \%$ & 65 & 45 \\
\hline $40-49$ & $0,35 \%$ & $9,96 \%$ & $6,97 \%$ & 29 & 20 \\
\hline $50-59$ & $0,80 \%$ & $10,82 \%$ & $9,09 \%$ & 14 & 11 \\
\hline $60-69$ & $1,24 \%$ & $14,12 \%$ & $10,29 \%$ & 11 & 8 \\
\hline $70+$ & $2,81 \%$ & $14,48 \%$ & $17,49 \%$ & 5 & 6 \\
\hline ption: $14 \%$ o & zedCOVID- & trers are treat & are (RKI) & & \\
\hline
\end{tabular}

Analyzing these estimates separately for the two extreme groups defined above, i.e. primary consumers of public TV vs. those who regularly consume private TV (but public TV not on a daily basis), much higher overestimations and risk estimates are found in the group of public TV viewers [11.8\% vs. $7.7 \%$ in the youngest group (18-29 years), $19.9 \%$ vs. $5.7 \%$ in the $70+$ group].

Using the QCOVID risk calculator from the University of Oxford (UK) (https://qcovid.org/Calculation) the individual severe morbidity (hospitalization) and mortality risk due to COVID-19 can be calculated even more accurately than shown in the table above (for more details see 1]. Just to give a few examples for different age groups and medical pre-conditions:

- 20-year old healthy male: $1: 33.333(0.003 \%)$ for severe disease, 1:1.000.000 (0.0001\%) for mortality

- 30-year old healthy female: 1:5.102 (0.021\%) for severe disease, 1:200.000 (0.004\%) for risk of death

- $\quad$ 40-year old healthy male: 1:3.300 (0.031\%) for severe disease, $1: 66.667(0.002 \%)$ for risk of death

- 50-year old overweight woman (BMI 28) with type II diabetes: 1:960 (0.037\%) for severe disease, $1: 6.536(0.003 \%)$ for risk of death

- 60-year old man with COPD: 1:738 (0.091\%) for severe disease, 1:4.274 (0.018\%) for risk of death.

In summary, the risk of dying from Covid-19 for individuals under 65 is extremely low and can be 
equated with the risk of having a fatal accident on the daily trip to work by car [3]. In this context we eagerly await that an unemotional discussion of "lockdown" measures - that have been enforced to varying degrees around the world [4] - is not only increasingly enforced in the scientific field but even more so appreciated in the public discussion and politics.

\section{Vaccination \& Testing Strategy}

In terms of willingness to be vaccinated, $42.8 \%$ of participants said in February 2021 that they will ,definitely“ get vaccinated (4.9\% of whom do so because of job requirements), $29.6 \%$,,prefer to wait“ or are „still undecided“, and 27.5\% will „definitely not“ get vaccinated. Among those who are in favor of vaccination, half (49.5\%) say the primary reason for vaccination is "to be able to return to a normal life." „Protecting oneself“ (26.4\%) or „others“ $(24.1 \%)$ is less often cited as the driving factor. The majority of those who oppose vaccination $(53.2 \%)$ believe that ,the side effects of vaccination are not yet well enough known or researched“.

According to the Austrian ministry of health (21. July 2021) 65,60\% of the "vaccinable" population aged $12+(5.183 .408$ people) was vaccinated at least once, and $52,90 \%$ fully.

In January/February $202192.4 \%$ of those who already had a PCR (35.84\%) or antigen $(49,43 \%)$ test received a negative test result. $87.9 \%$ of those who received a positive test result reported „no“ or „mild symptoms“, and the remaining $12.1 \%$ reported severe symptoms without (10.53\%) or with hospitalisation (1.58\%). In Austria, a test for recovery or SARS-CoV-2 antibodies was only performed by $11.69 \%$ by February $19^{\text {th }}$ 2021. The latter is remarkable, as already in December 2020 seroprevalence was estimated to be around $20 \%$ in the general population $[5,6]$ and up to $42 \%$ following SARS-CoV-2 outbreaks like in Ischgl (Austria; March 2020) [7]. In the Austrian population, seroprevalence was however not widely tested even as phase 3 of the vaccination plan was launched in April and May 2021 with the start of vaccination among the general population.

\section{Return to Normality}

The fact that (in January/February 2021) 43.2\% of the participants do not expect a return to normality until 2022 or even later can be interpreted as an alarming lack of perspective in the general population. Very similar and even more alarming results are found in the infant and adolescent population (aged 6-18) in Austria (cf. https://osf.io/fxamb/). The things most likely to help adult participants get through this crisis are „spending time in nature“ (76.4\%), „sports and exercise“ (72\%), and ,meeting relatives or friends in person“ $(68.1 \%)$.

These Austrian data $(n=3848)$, as well as the data from Germany $(n=3745)$ and Switzerland $(n=$ 
1815), can be accessed and visualized directly at bit.ly/CovidSurvey-DACH. It can be considered a 'work in progress' database where data should be accessible and widely shared with the broad public. In the following section, the data of 657 University students from Austria (aged 18-39 years) were evaluated separately in order to better understand their perspective on the pandemic and the potential stress and burden they experienced as also their daily life changed dramatically with now 3 semesters being abandoned in-presence, and practically all the teaching being shifted to a digital format.

\section{Spotlight on University Students in Austria}

In a special evaluation for students of the study "Knowledge and Attitudes Towards Corona", the data of a total of 657 students from Austria were analyzed (67.0 female, $32.1 \%$ male, $0.9 \%$ diverse). This survey closed May $9^{\text {th }} 2021$. Of these, $85.1 \%$ were in the $18-29$ age group and $14.9 \%$ in the 30-39 group.

90.1\% of students in Austria feel „very“ (61.5\%) or at least „somewhat“ (28.6\%) restricted by the Corona-related measures. A very large number of students share these concerns/displeasure in private with friends or family ,regularly“ or „,several times“ (76.5\%) However, only $10.1 \%$ also engage publicly by posting in forums, participating in demonstrations, or taking legal action. About a quarter of participants $(23 \%)$ were bothered ,all the time“ or ,most of the time“ by feelings of anger and unease due to the impression of non-objective reports.

$45.9 \%$ of students feel "very“ $(9.9 \%)$ or „somewhat“ (36.0\%) threatened by the coronavirus pandemic. In comparison, many more namely $55.7 \%$ of German students in the survey $(n=158)$ say they feel ,very“ (26.6\%) or „somewhat“ (29.1\%) threatened by the corona pandemic.

The greatest fears perceived by students in the pandemic are 1) that a close relative could get infected (76.2\%) 2) the long-term physical consequences (48.6\%) and 3) the psychological damage (44.6\%) expected. On the other hand, the fear of actually dying from Corona was least frequently mentioned $(12.8 \%)$.

What causes the students most stress is 1$)$ not being able to maintain social contacts $(52.4 \%$ not being able to meet friends, $44.3 \%$ not being able to meet relatives), 2) the economic consequences $(31.7 \%)$ and 3 ) the fear that a close relative may get infected (30.1\%). Only 3.8\% said they were concerned about getting sick themselves.

Regarding the willingness to be vaccinated, $43.2 \%$ of the student population stated that they will „definitely“ get vaccinated, 36.2\% would „like to wait“" or are „still undecided“, and 14.9\% will „definitely not“" get vaccinated. Compared to German students, the willingness to get vaccinated is 
significantly higher among Austrian students. According to the survey, only $24.7 \%$ of German students will „definitely“ get vaccinated and 43.7\% will „definitely not“ get vaccinated. However, it should be critically noted that the German student sample is probably too small in order to allow a reliable interpretation of this intercultural difference to date.

208 Among those in favor of vaccination, almost half of the students (49.2\%) say that the primary reason for vaccination is "to be able to return to a normal life" and $34.8 \%$ are in favor of vaccination ,to protect others“. Wanting to protect themselves from a Covid-19 disease is cited by only $16 \%$ as the

211 driving factor for a SARS-CoV-2 vaccination.

212 For the majority of those who oppose vaccination $(63.1 \%)$ the main reason is ,that the side effects of 213 vaccination are not well known or investigated enough“.

$21490.1 \%$ of those who already had a PCR or antigen test received a negative test result. $85.1 \%$ of those 215 who received a positive test result reported „no“ or „mild symptoms“, and the remaining $14.9 \%$ reported "severe symptoms". A test for SARS-CoV-2 recovery or already existing antibodies has been performed by only $10.5 \%$ of the Austrian and $10.8 \%$ of the German students by May 2021 .

Also a big part of the student population expects a return to normality only in 2022 or even later (41.7\%). Last but not least, students were asked what will best help them through the Corona crisis. They answer in the first 3 priorities with: 1) „meeting friends and loved ones in person“ $(67 \%), 2)$ „enjoying time in nature“ (66\%) and 3) „sports and exercise“ (62\%).

\section{Conclusion}

Altogether the results of this big (online) survey in German-speaking countries (here with a focus on Austria) reflects the high degree of the psychological burden and anxiety regarding SARS$\mathrm{CoV}-2$ in the general population. The subjectively estimated threat of the disease (hospitalisation or mortality) is vastly overestimated and contributes to the degree of psychosocial burdens in the Austrian population. As such, psychosocial effects are also known to have long-term consequences on the immune system and general health, it is highly advisable to quickly return to an objective 230 discussion of real risks and establish countermeasures to ease the psychosocial burden for those in need of support. The aftermath of the pandemic is just beginning and the public focus should finally be turned to those indirectly harmed by the coronavirus measures in order to prevent a meltdown of well-being and general somatic as well as mental health in the general population. 
References:

1. Florea C, Topalidis P, Hauser T, Angerer M, Kurapov A, Beltran Leon CA, Soares Brandão D, Schabus M. Sleep during COVID-19 lockdown: A cross-cultural study investigating job system relevance. Biochem Pharmacol. 2021 Feb 10:114463. doi: 10.1016/j.bcp.2021.114463. Epub ahead of print. PMID: 33577893; PMCID: PMC7872856.

2. Clift AK, Coupland CAC, Keogh RH, Diaz-Ordaz K, Williamson E, Harrison EM, Hayward A, Hemingway H, Horby P, Mehta N, Benger J, Khunti K, Spiegelhalter D, Sheikh A, Valabhji J, Lyons RA, Robson J, Semple MG, Kee F, Johnson P, Jebb S, Williams T, Hippisley-Cox J. Living risk prediction algorithm (QCOVID) for risk of hospital admission and mortality from coronavirus 19 in adults: national derivation and validation cohort study. BMJ.

2020 Oct 20;371:m3731. doi: 10.1136/bmj.m3731. PMID: 33082154; PMCID: PMC7574532.

3. Ioannidis JPA, Axfors C, Contopoulos-Ioannidis DG. Population-level COVID-19 mortality risk for non-elderly individuals overall and for non-elderly individuals without underlying diseases in pandemic epicenters. Environ Res. 2020 Sep;188:109890. doi: 10.1016/j.envres.2020.109890.epub 2020 Jul 1. PMID: 32846654; PMCID:PMC7327471.

4. Melnick ER, Ioannidis JPA. Should governments continue lockdown to slow the spread of covid-19? BMJ.2020 Jun 3;369:m1924. doi: 10.1136/bmj.m1924. PMID:32493767.

5. Mortgat L, Verdonck K, Hutse V, Thomas I, Barbezange C, Heyndrickx L, Fischer N, Vuylsteke B, Kabouche I, Ariën KK, Desombere I, Duysburgh E. Prevalence and incidence of anti-SARS-CoV-2 antibodies among healthcare workers in Belgian hospitals before vaccination: a prospective cohort study. BMJ Open. 2021 Jun 29;11(6):e050824. doi: 10.1136/bmjopen-2021-050824. PMID: 34187832; PMCID: PMC8245288.

6. Stringhini S, Zaballa ME, Perez-Saez J, Pullen N, de Mestral C, Picazio A, Pennacchio F, Wisniak A, Richard A, Baysson H, Loizeau A, Balavoine JF, Trono D, Pittet D, PosfayBarbe K, Flahault A, Chappuis F, Kherad O, Vuilleumier N, Kaiser L, Azman AS, Guessous I; Specchio-COVID19 Study Group. Seroprevalence of anti-SARS-CoV-2 antibodies after the second pandemic peak. Lancet Infect Dis. 2021 May;21(5):600-601. doi: 10.1016/S1473-3099(21)00054-2. Epub 2021 Feb 1. PMID: 33539733; PMCID: PMC8063076.

7. Knabl, L., Mitra, T., Kimpel, J. et al. High SARS-CoV-2 seroprevalence in children and adults in the Austrian ski resort of Ischgl. Commun Med 1, 4 (2021). https://doi.org/10.1038/s43856021-00007-1 